\title{
Identification of Underlying Partial Differential Equations from Noisy Data with Splines
}

\author{
Yujie Zhao, Xiaoming Huo (presenting author), and Yajun Mei \\ H. Milton Stewart School of Industrial and Systems Engineering, \\ Georgia Tech, USA
}

\begin{abstract}
We propose a two-stage method called Spline Assisted Partial Differential Equation involved Model Identification (SAPDEMI) to efficiently identify the underlying partial differential equation (PDE) models from the noisy data. In the first stage, we employ the cubic spline to estimate the unobservable derivatives, where some of them govern the underlying PDE models. This stage is computationally efficient, i.e., it only requires the computational complexity of the linear polynomial of the sample size, which achieves the lowest possible order of complexity. In the second stage, we apply Least Absolute Shrinkage and Selection Operator (Lasso) to identify the underlying PDE models, where we focus on the model selections, instead of parameter estimations in the existing literature. Moreover, we develop statistical properties of our method for the correct identification. Finally, we validate our theory through various numerical examples and apply it to a case study analyzing the data downloaded from the National Aeronautics and Space Administration (NASA).
\end{abstract}

